American Journal of Applied Sciences 5 (11): 1461-1466, 2008

ISSN 1549-3644

(C) 2008 Science Publications

\title{
An Order Six Zero-Stable Method for Direct Solution of Fourth Order Ordinary Differential Equations
}

\author{
Kayode Sunday Jacob \\ Department of Mathematical Sciences \\ Federal University of Technology, P.M.B 704, Akure, Nigeria
}

\begin{abstract}
A zero-stable numerical method for direct solution of fourth order differential equations reduces the computational burden and computer time wastage involved in the method of reducing such equations to a system of first order equations. The method adopted is the collocation of the differential system and the interpolation of the approximate solution to the problem using the power series as a basis function. The method is consistent and symmetric with optimal order $p=6$. A consistent and symmetric main predictor of order five is also developed for the evaluation of the implicit scheme.
\end{abstract}

Keywords: Zero-stable, collocation, continuous coefficient, optimal order, functions evaluation.

\section{INTRODUCTION}

The empirical problems leading to higher order differential equations of the form

$$
y^{(m)}=f\left(t, y, y^{1}, y^{2}, \ldots, y^{(m-1)}\right), y^{(m-1)}\left(t_{0}\right)=\mu_{m-1}, m=1,2, \ldots
$$

are often encountered especially by Scientists and Engineers. The solutions of such equations have engaged the attention of many mathematicians, both the theorists and numerical analysts. Many of such empirical results yielding higher order differential equations are not solvable analytically. Numerical methods adopted for such higher order differential equations are only capable of handling first order equations of type

$y^{\prime}=f(t, y), y\left(t_{0}\right)=\mu, f \in C[a, b], y, t \in R^{n}$.

This implies that such problems will be reduced to system of first order equations ${ }^{[1],[8],[10]}$. The approach of reducing such equations to a system of first order equations leads to serious computational burden and wastage in computer time ${ }^{[2],[3]}$.

Many attempts have been made to formulate numerical algorithms capable of solving special problem of type (1) without reducing it to system of first order equations ${ }^{[7],}[9],[11],[12]$. Multiderivative methods for direct solution of problem (1), taking $\mathrm{m}=$ 4 , have been considered using canonical polynomial as the basis functions ${ }^{[4],[5]}$. They produced methods with many function evaluations.

Efforts are made to develop a zero-stable order six method of higher step-number with reduced functions evaluation for direct solution of problem (1) for $\mathrm{m}=4$. The reduced functions evaluation of this method further lessened the computational burden ${ }^{[3],[6]}$.

\section{METHODS OF SOLUTION}

The proposed zero-stable numerical method of order six for direct solution of fourth order differential equations is of the form of a continuous linear multistep

$$
y_{k}(x)=\sum_{j=0}^{k-1} \alpha_{j}(x) y_{n+j}+h^{4} \sum_{j=2}^{k} \beta_{j}(x) f_{n+j}
$$

Let the approximate solution $y(x)$ to problem (1) be taken to be a partial sum of a power series $\varphi_{j}(x)$ of a single variable $\mathrm{x}$ in the form

$\varphi_{j}(x)=\sum_{j=0}^{2(k-1)} a_{j} x^{j}$

where $a_{j}{ }^{\prime} s, j=0,1, \ldots, 2(k-1)$, are real coefficients.

The first, second and third order derivatives of

(4a) are respectively given as 
American J. App. Sc., 5 (11): 1461-1466, 2008

$$
\begin{aligned}
& \varphi_{j}^{(1)}(x)=\sum_{j=1}^{2(K-1)} j a_{j} x^{j-1} \\
& \varphi_{j}^{(2)}(x)=\sum_{j=2}^{2(k-1)} j(j-1) a_{j} x^{j-2} \\
& \varphi_{j}^{(3)}(x)=\sum_{j=3}^{2(k-1)} j(j-1)(j-2) a_{j} x^{j-3} \\
& \varphi_{j}^{(4)}(x)=\sum_{j=4}^{2(k-1)} j(j-1)(j-2)(j-3) a_{j} x^{j-4} \\
& \sum_{j=4}^{2(k-1)} j(j-1)(j-2)(j-3) a_{j} x^{j-4}=f\left(x, y, y^{(1)}, y^{(2)}, y^{(3)}\right)
\end{aligned}
$$

Equation (5) was collocated at some selected grid points $x=x_{n+i}, i=1,3, \ldots, k$, while equation (4a) was interpolated at grid points $x=x_{n+i}, i=0,1, \ldots, k-1$, to have a system of linear equations

$$
\begin{aligned}
& \sum_{j=4}^{2(k-1)} j(j-1)(j-2)(j-3) a_{j} \varphi_{j-4}\left(x_{n+i}\right)=f_{n+i}, i=1,3, \ldots, k \\
& \sum_{j=0}^{2(k-1)} a_{j} \varphi_{j}\left(x_{n+i}\right)=y_{n+i}, i=0,1, \ldots, k-1
\end{aligned}
$$

where

$$
\begin{aligned}
& f_{n+i}=f\left(x_{n+i}, y_{n+i}, y_{n+i}^{(1)}, y_{n+i}^{(2)}, y_{n+i}^{(3)}\right), i=0,1,2, \ldots, k \\
& y_{n+i}=y\left(x_{n+i}\right) \text { and } y^{(r)}=\frac{d^{r} y}{d x^{r}} .
\end{aligned}
$$

Solving the system of equations (6), (7) for $a_{j}^{\prime} s$, $j=0,1, \ldots, k+2$ and substituting the results into (4a), we obtained a continuous method of type (3).

Using the transformation ${ }^{[6]}$

$x=t h+x_{n+4}, t \in(0,1]$,

equation (3) translates to

$$
y_{k}(t)=\sum_{j=0}^{k-1} \alpha_{j}(t) y_{n+j}+\sum_{j=0}^{k} \beta_{j}(t) f_{n+j}
$$

The coefficients $\alpha_{j}(t)$ and $\beta_{j}(t)$ obtained as $\alpha_{0}(t)=\frac{1}{1680}\left(132 t+98 t^{2}-126 t^{3}-105 t^{4}-7 t^{5}+7 t^{6}+t^{7}\right)$,

$$
\begin{aligned}
& \alpha_{1}(t)=-\frac{1}{420}\left(272 t+308 t^{2}-56 t^{3}-105 t^{4}-7 t^{5}+7 t^{6}+t^{7}\right), \\
& \alpha_{2}(t)=\frac{1}{280}\left(552 t+658 t^{2}+14 t^{3}-105 t^{4}-7 t^{5}+7 t^{6}+t^{7}\right), \\
& \alpha_{3}(t)=-\frac{1}{420}\left(1392+1148^{2}+84 t^{3}-105^{4}-7 t^{5}+7 t^{6}+t^{7}\right), \\
& \alpha_{4}(t)=\frac{1}{1680}\left(1680+3212 t+1778 t^{2}+154 t^{3}-105 t^{4}-7 t^{5}+7 t^{6}+t^{7}\right), \\
& \beta_{1}(t)=\frac{1}{20160}\left(-240 t+284 t^{2}+630 t^{3}+420 t^{4}+35 t^{5}-28 t^{6}-5 t^{7}\right), \\
& \beta_{3}(t)=\frac{1}{10080}\left(1824 t+3920^{2}+2814 t^{3}+73 t^{4}-14 t^{5}-35 t^{6}-4 t^{7}\right), \\
& \beta_{5}(t)=\frac{1}{20160}\left(48 t+196 t^{2}+294 t^{3}+210 t^{4}+77 t^{5}+14 t^{6}+t^{7}\right) .
\end{aligned}
$$

The first, second and third order derivatives of (9) were computed and given as follows:

Fist derivative of (10):

$$
\begin{aligned}
& \alpha_{0}^{\prime}(t)=\frac{1}{1680 h}\left(132+196 t-378 t^{2}-420 t^{3}-35 t^{4}+42 t^{5}+7 t^{6}\right), \\
& \alpha_{1}^{\prime}(t)=\frac{-1}{420 h}\left(272+616 t-168 t^{2}-420 t^{3}-35 t^{4}+42 t^{5}+7 t^{6}\right), \\
& \alpha_{2}^{\prime}(t)=\frac{1}{280 h}\left(552+1316 t+42 t^{2}-420 t^{3}-35 t^{4}+42 t^{5}+7 t^{6}\right), \\
& \alpha_{3}^{\prime}(t)=-\frac{1}{420 h}\left(1392+2296 t+252 t^{2}-420 t^{3}-35 t^{4}+42 t^{5}+7 t^{6}\right), \\
& \alpha_{4}^{\prime}(t)=\frac{1}{1680 h}\left(3212+3556 t+462 t^{2}-420 t^{3}-35 t^{4}+42 t^{5}+7 t^{6}\right), \\
& \beta_{1}^{\prime}(t)=\frac{1}{20160 h}\left(-240+56 t+1890 t^{2}+1680 t^{3}+175 t^{4}-168 t^{5}-35 t^{6}\right), \\
& \beta_{3}^{\prime}(t)=\frac{1}{10080 h}\left(1824+7820 t+8442 t^{2}+2940 t^{3}-70 t^{4}-210 t^{5}-28 t^{6}\right), \\
& \beta_{5}^{\prime}(t)=\frac{1}{20160 h}\left(48+392 t+882 t^{2}+840 t^{3}+385 t^{4}+84 t^{5}+7 t^{6}\right),
\end{aligned}
$$

Second derivative

$$
\begin{aligned}
& \alpha_{0}^{\prime \prime}(t)=\frac{1}{1680 h^{2}}\left(196-756 t-1260 t^{2}-140 t^{3}+210 t^{4}+42 t^{5}\right), \\
& \alpha_{1}^{\prime \prime}(t)=-\frac{1}{420 h^{2}}\left(616-336 t-1260 t^{2}-140 t^{3}+210 t^{4}+42 t^{5}\right), \\
& \alpha_{2}^{\prime \prime}(t)=\frac{1}{280 h^{2}}\left(1316+84 t-1260 t^{2}-140 t^{3}+210 t^{4}+42 t^{5}\right), \\
& \alpha_{3}^{\prime \prime}(t)=-\frac{1}{420 h^{2}}\left(2296504 t-1260 t^{2}-140 t^{3}+210 t^{4}+42 t^{5}\right), \\
& \alpha_{4}^{\prime \prime}(t)=\frac{1}{1680 h^{2}}\left(3556924-1260^{2}-140^{3}+210^{4}+42 t^{5}\right),
\end{aligned}
$$




$$
\begin{aligned}
& \beta_{1}^{\prime \prime}(t)=\frac{1}{20160 h^{2}}\left(56+3780 t+5040 t^{2}+700 t^{3}-840 t^{4}-210 t^{5}\right), \\
& \beta_{3}^{\prime \prime}(t)=\frac{1}{10080 h^{2}}\left(7820+16884 t+8820 t^{2}-280 t^{3}-1050 t^{4}-168 t^{5}\right), \\
& \beta_{5}^{\prime \prime}(t)=\frac{1}{20160 h^{2}}\left(392+1764 t+2520 t^{2}+1540 t^{3} 420 t^{4}+42 t^{5}\right) .
\end{aligned}
$$

Third derivative

$$
\begin{aligned}
& \alpha_{0}^{\prime \prime \prime}(t)=\frac{1}{1680 h^{3}}\left(-756-2520 t-420 t^{2}+840 t^{3}+210 t^{4}\right), \\
& \alpha_{1}^{\prime \prime \prime}(t)=-\frac{1}{420 h^{3}}\left(-336-2520-420 t^{2}+840 t^{3}+210 t^{4}\right) \\
& \alpha_{2}^{\prime \prime \prime}(t)=\frac{1}{280 h^{3}}\left(84-2520-420 t^{2}+840 t^{3}+210 t^{4}\right) \\
& \alpha_{3}^{\prime \prime \prime}(t)=-\frac{1}{420 h^{3}}\left(5044-2520-420 t^{2}+840 t^{3}+210 t^{4}\right) \\
& \alpha_{3}^{\prime \prime \prime}(t)=-\frac{1}{420 h^{3}}\left(5044-2520-420^{2}+840^{3}+210^{4}\right) \\
& \alpha_{4}^{\prime \prime \prime}(t)=\frac{1}{1680 h^{3}}\left(924-2520-420 t^{2}+840 t^{3}+210 t^{4}\right) \\
& \beta_{1}^{\prime \prime \prime}(t)=\frac{1}{20160 h^{4}}\left(3780+10080 t+2100 t^{2}-3360 t^{3}-1050 t^{4}\right) \\
& \beta_{3}^{\prime \prime \prime}(t)=\frac{1}{10080 h^{4}}\left(16884+17640 t+2100 t^{2}-3360 t^{3}-1050 t^{4}\right) \\
& \beta_{5}^{\prime \prime \prime}(t)=\frac{1}{20160 h^{4}}\left(1764+5040 t+4620 t^{2}+1680 t^{3}+2100 t^{4}\right)
\end{aligned}
$$

For any sample discrete scheme to be determined from the continuous method (9) and its first, second and third derivatives respectively, the values of $t$ could be taken in the interval $I=(0,1]$. In this work, the value of $t$ was taken to be 1 to obtain a zero-stable discrete method and its derivatives as

$$
y_{n+5}=4 y_{n+4}-6 y_{n+3}+4 y_{n+2}-y_{n+1}+\frac{h^{4}}{24}\left(f_{n+5}+22 f_{n+3}+f_{n+1}\right)
$$

$y_{n+5}^{(1)}=\frac{1}{210 h}\left\{853 y_{n+4}-1767 y_{n+3}+1128 y_{n+2}-157 y_{n+1}-57 y_{n}\right.$

$\left.+\frac{h^{4}}{48}\left(1319 f_{n+5}+20738 f_{n+3}+1679 f_{n+1}\right)\right\}$

$y_{n+5}^{(2)}=\frac{1}{60 h^{2}}\left\{119 y_{n+4}-236 y_{n+3}+54 y_{n+2}+124 y_{n+1}-60 y_{n}\right.$

$\left.+\frac{h^{4}}{8}\left(159 f_{n+5}+1526 f_{n+3}+203 f_{n+1}\right)\right\}$ $y_{n+5}^{(3)}=\frac{1}{40 h^{3}}\left\{-23 y_{n+4}+132 y_{n+3}-258 y_{n+2}+212 y_{n+1}-63 y_{n}\right.$

$\left.+\frac{h^{4}}{12}\left(317 f_{n+5}+1364 f_{n+3}+275 f_{n+1}\right)\right\}$

Using the local truncation error approach ${ }^{[13]}$, the order $\mathrm{P}$ and error constant $\mathrm{C}_{\mathrm{p}+2}$ of method (14) were found to be 6 and $-\frac{31}{720}$ respectively.

The discrete method (14) was found to be consistent and zero stable, satisfying the necessary and sufficient conditions for the convergence of Linear Multistep Methods ${ }^{[9],[13]}$.

\section{IMPLIMENTATION OF THE METHOD}

The continuous method (9) was designed to solve general differential equations of type (1) where the collocated $f_{n+i}$ has been defined as

$f_{n+i}=f\left(x_{n+i}, y_{n+i}, y_{n+i}^{(1)}, y_{n+i}^{(2)}, y_{n+i}^{(3)}\right), i=0,1,2, \ldots, k$.

By definition, the method is implicit if $\beta_{k} \neq 0$ and explicit if otherwise ${ }^{[13]}$. To implement the sample discrete method (14) and its derivatives, additional explicit starting values for $y_{n+i}, y_{n+i}^{(r)}, \mathrm{i}=1,2 \ldots, \mathrm{k}-1$, were obtained by using the same approach described in equations (3) through (8) to obtain the following consistent and zero stable explicit methods

$y_{k}(t)=\sum_{j=0}^{k-1} \alpha_{j}(t) y_{n+j}+\sum_{j=0}^{k-1} \beta_{j}(t) f_{n+j}$,

where $k=5,4$.

For $\mathrm{k}=5$ :

$y_{n+5}=4 y_{n+4}-6 y_{n+3}+4 y_{n+2}-y_{n+1}+\frac{h^{4}}{12}\left(5 f_{n+4}+8 f_{n+2}-f_{n}\right)$,

$\mathrm{p}=5, C_{p+2} \approx-\frac{1}{3}$,

$y_{n+5}^{(1)}=\frac{1}{6 h}\left\{26 y_{n+4}-57 y_{n+3}+42 y_{n+2}-11 y_{n+1}\right.$

$\left.+\frac{h^{4}}{120}\left(659 f_{n+4}+904 f_{n+2}-13 f_{n}\right)\right\}$

$\mathrm{p}=5, C_{p+2} \approx-\frac{2473}{4000}$, 


$$
\begin{aligned}
& y_{n+5}^{(2)}=\frac{1}{h^{2}}\left\{3 y_{n+4}-8 y_{n+3}+7 y_{n+2}-2 y_{n+1}\right. \\
& \left.+\frac{h^{4}}{720}\left(1237 f_{n+4}+976 f_{n+2}-113 f_{n}\right)\right\} \\
& \mathrm{p}=5, C_{p+2} \approx-\frac{11}{20}, \\
& y_{n+5}^{(3)}=\frac{1}{h^{3}}\left\{y_{n+4}-3 y_{n+3}+3 y_{n+2}-y_{n+1}\right. \\
& \left.+\frac{h^{4}}{24}\left(52 f_{n+4}+7 f_{n+2}+f_{n}\right)\right\} \\
& \mathrm{p}=5, C_{p+2} \approx-\frac{31}{60} .
\end{aligned}
$$

For $\mathrm{k}=4$ :

$$
\begin{aligned}
& y_{n+4}=4 y_{n+3}-6 y_{n+2}+4 y_{n+1}-y_{n} \\
& +\frac{h^{4}}{6}\left(f_{n+3}+4 f_{n+2}+f_{n+1}\right) \\
& \mathrm{p}=6, C_{p+2} \approx-\frac{1}{720}, \\
& y_{n+4}^{(1)}=\frac{1}{6 h}\left\{26 y_{n+3}-57 y_{n+2}+42 y_{n+1}-11 y_{n}\right. \\
& \left.+\frac{h^{4}}{60}\left(185 f_{n+3}+452 f_{n+2}+113 f_{n+1}\right)\right\} \\
& \mathrm{p}=5, C_{p+2} \approx \frac{1}{105} \text {, } \\
& y_{n+4}^{(2)}=\frac{1}{h^{2}}\left\{3 y_{n+3}-8 y_{n+2}+7 y_{n+1}-2 y_{n}\right. \\
& \left.+\frac{h^{4}}{60}\left(449 f_{n+3}+452 f_{n+2}+149 f_{n+1}\right)\right\} \\
& \mathrm{p}=5, C_{p+2} \approx \frac{7}{90} \\
& y_{n+4}^{(3)}=\frac{1}{h^{3}}\left\{y_{n+3}-3 y_{n+2}+3 y_{n+1}-y_{n}\right. \text {. } \\
& \left.+\frac{h^{4}}{24}\left(55 f_{n+4}-8 f_{n+2}+13 f_{n+1}\right)\right\} \\
& \mathrm{p}=5, C_{p+2} \approx \frac{7}{20}
\end{aligned}
$$

The minimum value of $\mathrm{k}$ for the development of any Linear Multistep Method must be equal to the order of the differential equation it is meant to solve. For each of $y_{n+i}, y_{n+i}^{(1)}, y_{n+i}^{(2)}, y_{n+i}^{(3)}, y_{n+i}, i=1, \ldots, k, k<4$,

Taylor series expansion was used to evaluate, to have

$$
\begin{aligned}
& y_{n+i} \equiv y\left(x_{n}+i h\right) \cong y\left(x_{n}\right)+i h y^{(1)}\left(x_{n}\right)+\frac{(i h)^{2}}{2 !} y^{(2)}\left(x_{n}\right) \\
& +\frac{(i h)^{3}}{3 !} y^{(3)}\left(x_{n}\right)+\frac{(i h)^{4}}{4 !} f_{n}+\frac{(i h)^{5}}{5 !} f_{n}^{(1)}+\ldots \\
& y^{(1)}{ }_{n+i} \equiv y^{(1)}\left(x_{n}+i h\right) \cong y^{(1)}\left(x_{n}\right)+i h y^{(2)}\left(x_{n}\right)+\frac{(i h)^{2}}{2 !} y^{(3)}\left(x_{n}\right) \\
& +\frac{(i h)^{3}}{3 !} f_{n}+\frac{(i h)^{4}}{4 !} f_{n}^{(1)}+\frac{(i h)^{5}}{5 !} f_{n}^{(2)}+\ldots
\end{aligned}
$$

$y_{n+i}^{(2)} \equiv y^{(2)}\left(x_{n}+i h\right) \cong y^{(2)}\left(x_{n}\right)+i h y^{(3)}\left(x_{n}\right)+\frac{(i h)^{2}}{2 !} f_{n}$

$+\frac{(i h)^{3}}{3 !} f_{n}^{(1)}+\frac{(i h)^{4}}{4 !} f_{n}^{(2)}+\frac{(i h)^{5}}{5 !} f_{n}^{(3)}+\ldots$

$y^{(3)}{ }_{n+i} \equiv y^{(3)}\left(x_{n}+i h\right) \cong y^{(3)}\left(x_{n}\right)+i h f$

$+\frac{(i h)^{2}}{2 !} f_{n}^{(1)}+\frac{(i h)^{3}}{3 !} f_{n}^{(2)}+\frac{(i h)^{4}}{4 !} f_{n}^{(3)}+\ldots$ Order $\mathrm{p}=5, C_{p+2} \approx \frac{1}{10}$

For $\mathrm{m}=4$ in equation (1) and taking $y^{(4)}\left(x_{n}\right)=f_{n}$,

$y_{n}^{(4+j)}=f_{n}^{(j)}, j=0,1,2, \ldots, f_{n}^{(0)}=f_{n}$ and

$f_{n}^{(j)}=f^{(j)}\left(x_{n}, y_{n}, y_{n}^{(1)}, y_{n}^{(2)}, y_{n}^{(3)}\right)$, the values of

$f_{n}^{(1)}, f_{n}^{(2)}$ were obtained by partial derivative technique as

$\left.f^{(1)}=\frac{d f}{d x}=\frac{\partial f}{\partial x}+y^{(1)} \frac{\partial f}{\partial y}+y^{(2)} \frac{\partial f}{\partial y^{(1)}}+y^{(3)} \frac{\partial f}{\partial y^{(2)}}+f \frac{\partial f}{\partial y^{(3)}}\right)$
$f^{(2)}=\frac{d^{2} f}{d x^{2}}=2\left(A y^{(1)}+B y^{(2)}+C y^{(3)}+D f+E+F\right.$,

where

$$
\begin{aligned}
& A=\frac{\partial^{2} f}{\partial x \partial y}+y^{(2)} \frac{\partial^{2} f}{\partial y \partial y^{(1)}}+y^{(3)} \frac{\partial^{2} f}{\partial y \partial y^{(2)}}+f \frac{\partial^{2} f}{\partial y \partial y^{(3)}} \\
& B=\frac{\partial^{2} f}{\partial x \partial y}+y^{(3)} \frac{\partial^{2} f}{\partial y^{(1)} \partial y^{(2)}}+f \frac{\partial^{2} f}{\partial y^{(1)} \partial y^{(3)}}, \\
& C=\frac{\partial^{2} f}{\partial x \partial y^{(2)}}+f \frac{\partial^{2} f}{\partial y^{(2)} \partial y^{(3)}}, \\
& D=\frac{\partial^{2} f}{\partial x \partial y^{(3)}}, \\
& E=y^{(2)} \frac{\partial f}{\partial y}+y^{(3)} \frac{\partial f}{\partial y^{(1)}}+f \frac{\partial f}{\partial y^{(2)}}+f^{\prime} \frac{\partial f}{\partial y^{(3)}} \\
& F=\frac{\partial^{2} f}{\partial x^{2}}+\left(y^{(1)}\right)^{2} \frac{\partial^{2} f}{\partial y^{2}}+\left(y^{(2)}\right)^{2} \frac{\partial^{2} f}{\left(\partial y^{(1)}\right)^{2}} \\
& +\left(y^{(3)}\right)^{2} \frac{\partial^{2} f}{\left(\partial y^{(2)}\right)}+(f)^{2} \frac{\partial^{2} f}{\left(\partial y^{(3)}\right)^{2}} .
\end{aligned}
$$

$y_{0}, y_{0}^{(1)}, y_{0}^{(2)}, y_{0}^{(3)}$ are the initial values given in the problems.

\section{SAMPLE TESTS AND RESULTS}

The accuracy of the method (14) was tested with two test problems and their results compared with existing method ${ }^{[6]}$ as shown in the tables below.

Test Problem 1:

$$
y^{(4)}+y^{(2)}=0,0 \leq x \leq \frac{\pi}{2},
$$


American J. App. Sc., 5 (11): 1461-1466, 2008

$$
\begin{aligned}
& y(0)=0, y^{(1)}(0)=\frac{-1.1}{72-50 \pi}, \\
& y^{(2)}(0)=\frac{1}{144-100 \pi}, \quad y^{(3)}(0)=\frac{1.2}{144-100 \pi} .
\end{aligned}
$$

$$
\begin{aligned}
& y^{(4)}=\left(y^{(1)}\right)^{2}-y y^{(3)}-4 x^{2}+e^{x}\left(1-4 x+x^{2}\right), \\
& 0 \leq x \leq 1, y(0)=1, y^{(1)}(0)=1, y^{(2)}(0)=3, y^{(3)}(0)=1 . \\
& \text { Theoretical solution: } \quad y(x)=x^{2}+e^{x} .
\end{aligned}
$$

Theoretical solution:

$$
y(x)=\frac{(1-x-\cos x-1.2 \sin x)}{(144-100 \pi)}
$$

Test Problem 2:

Table 1: Comparison of errors arising from Awoyemi and Kayode ${ }^{[6]}$ and New Method (14) for Problem 1

\begin{tabular}{ccccc}
\hline $\mathrm{X}$ & Exact solution & Computed Method (14) & $\begin{array}{c}\text { Awoyemi and } \\
\text { Kayode }^{[6]}\end{array}$ & Error in Method (14) \\
\hline 0.103125 & $0.130079934027 \mathrm{D}-02$ & $0.130079934027 \mathrm{D}-02$ & $0.215807406667 \mathrm{D}-12$ & $0.498732999343 \mathrm{D}-15$ \\
0.206250 & $0.253177321538 \mathrm{D}-02$ & $0.253177321538 \mathrm{D}-02$ & $0.235634428636 \mathrm{D}-12$ & $0.676542155631 \mathrm{D}-15$ \\
0.306250 & $0.365247827946 \mathrm{D}-02$ & $0.365247827947 \mathrm{D}-02$ & $0.250504044591 \mathrm{D}-12$ & $0.313507900196 \mathrm{D}-14$ \\
0.406250 & $0.469595233257 \mathrm{D}-02$ & $0.469595233258 \mathrm{D}-02$ & $0.258852835000 \mathrm{D}-12$ & $0.943602834758 \mathrm{D}-14$ \\
0.506250 & $0.565764127720 \mathrm{D}-02$ & $0.565764127722 \mathrm{D}-02$ & $0.257955115601 \mathrm{D}-12$ & $0.221168569570 \mathrm{D}-13$ \\
0.603125 & $0.650775336185 \mathrm{D}-02$ & $0.650775336190 \mathrm{D}-02$ & $0.245233520990 \mathrm{D}-12$ & $0.433793626020 \mathrm{D}-13$ \\
0.703125 & $0.729831337007 \mathrm{D}-02$ & $0.729831337015 \mathrm{D}-02$ & $0.216478744652 \mathrm{D}-12$ & $0.778708694749 \mathrm{D}-13$ \\
0.803125 & $0.799851869108 \mathrm{D}-02$ & $0.799851869121 \mathrm{D}-02$ & $0.168292463298 \mathrm{D}-12$ & $0.128634949914 \mathrm{D}-12$ \\
0.903125 & $0.860724505508 \mathrm{D}-02$ & $0.860724505528 \mathrm{D}-02$ & $0.969693075836 \mathrm{D}-13$ & $0.199271155132 \mathrm{D}-12$ \\
1.003125 & $0.912428221980 \mathrm{D}-02$ & $0.912428222010 \mathrm{D}-02$ & $0.893382590128 \mathrm{D}-15$ & $0.293232452209 \mathrm{D}-12$ \\
\hline
\end{tabular}

Table 3: Comparison of errors arising from Awoyemi and Kayode ${ }^{[6]}$ and New Method (14) for Problem 2

\begin{tabular}{ccccc}
\hline $\mathrm{X}$ & Exact solution & Computed Method (14) & $\begin{array}{c}\text { Awoyemi and } \\
\text { Kayode }^{[6]}\end{array}$ & Error in Method (14) \\
\hline 0.103125 & $0.111926474479 \mathrm{D}+01$ & $0.111926474479 \mathrm{D}+01$ & $0.817323986269 \mathrm{D}-11$ & $0.666133814775 \mathrm{D}-14$ \\
0.206250 & $0.127159949320 \mathrm{D}+01$ & $0.127159949320 \mathrm{D}+01$ & $0.788127341167 \mathrm{D}-09$ & $0.974775815621 \mathrm{D}-13$ \\
0.306250 & $0.145211090707 \mathrm{D}+01$ & $0.145211090706 \mathrm{D}+01$ & $0.146806344858 \mathrm{D}-09$ & $0.461852778244 \mathrm{D}-12$ \\
0.406250 & $0.166621686250 \mathrm{D}+01$ & $0.166621686250 \mathrm{D}+01$ & $0.267951016930 \mathrm{D}-08$ & $0.142241773915 \mathrm{D}-11$ \\
0.506250 & $0.191534710992 \mathrm{D}+01$ & $0.191534710992 \mathrm{D}+01$ & $0.706149827501 \mathrm{D}-08$ & $0.342548212018 \mathrm{D}-11$ \\
0.603125 & $0.219158159361 \mathrm{D}+01$ & $0.219158159360 \mathrm{D}+01$ & $0.154384078854 \mathrm{D}-07$ & $0.687361279006 \mathrm{D}-11$ \\
0.703125 & $0.251444029333 \mathrm{D}+01$ & $0.251444029332 \mathrm{D}+01$ & $0.309007592847 \mathrm{D}-07$ & $0.126365584663 \mathrm{D}-10$ \\
0.803125 & $0.287751638775 \mathrm{D}+01$ & $0.287751638773 \mathrm{D}+01$ & $0.567595508372 \mathrm{D}-07$ & $0.213882245248 \mathrm{D}-10$ \\
0.903125 & $0.328293615881 \mathrm{D}+01$ & $0.328293615877 \mathrm{D}+01$ & $0.975496026179 \mathrm{D}-07$ & $0.339896999435 \mathrm{D}-10$ \\
1.003125 & $0.373304951150 \mathrm{D}+01$ & $0.373304951144 \mathrm{D}+01$ & $0.158970819619 \mathrm{D}-06$ & $0.514028819509 \mathrm{D}-10$ \\
\hline
\end{tabular}

\section{RESULTS AND DISCUSSION}

Tables 1 and 2 above show the results of problems 1 and 2 respectively when solved with the new method (14). The maximum errors arising from the problems when solved with the existing methods ${ }^{[6]}$ for a mesh size $\mathrm{h}=1 / 32$ were compared with the errors of the new method (14) for the same mesh size as shown in the Tables.

\section{CONCLUSION}

The order six method with three functions evaluation 
developed through collocation approach is capable of solving linear and non-linear general fourth order ordinary differential equations directly without reduction to system of first order equations. This reduced the computational burden and its inevitable effects on computer time.

The method is consistent and zero stable, satisfying the basic requirements for convergence of Linear Multistep methods (LMM). All the predictors and their derivatives are consistent and zero stable. Efforts were made to ensure that the orders of the predictors and their derivatives are close to the order of the method in order to reduce the effects this could have on accuracy. While the order of accuracy of the new method is 6 , the orders of the predictors and their derivatives are 5 and 6 .

The zero stability property and the reduced functions evaluation as well as the comparative high order of the predictors of the new method (14) serve as advantages over the existing method ${ }^{[6]}$. In spite of its lower order, this comparative advantage probably accounts for better accuracy of the new method as shown in Tables 1 and 2.

\section{REFERENCES}

1. Abhulimen, C.E. and F.O. Otunta, 2006. A sixth order multiderivative multistep methods for stiff system of differential equations. Intern J. Num. Math., 1(2): $248-268$.

2. Awoyemi, D.O., 2001. A new sixth-order algorithm for general second order ordinary differential equations. Int. J. Comp. Math., 77: 117-124.

3. Awoyemi, D.O., 2005. Algorithmic collocation approach for direct solution of fourth order initial value problems of ordinary differential equations. Intl. J. Comp. Math. 82(3): 271- 284.
4. Awoyemi, D.O., 2003. A p-stable linear multistep method for the solution of genrral third order ordinary differential equations. Intl. J. Comp. Math. 80(8): 987-993.

5. Awoyemi, D.O. and S.J. Kayode, 2003. An optimal order collocation method for direct solution of initial value problems of general second order ordinary differential equations. J. Nig. Math Phys, 6: 285-292.

6. Awoyemi, D.O. and S.J. Kayode, 2004. Maximal order multiderivative collocation method for direct solution of fourth order initial value problems of ordinary differential equations. J. Nig. Math. Soc., 2 3: $53-64$.

7. Bun, R. A. and Y.D Vasil'Yev, 1992. A numerical method for solving differential equations of any orders. Comp. Math. Phys, 32(3): 317-330.

8. Chan, R. P. K, P. Leone and A. Tsai, 2004. Order conditions and symmetry for two-step hybrid methods. Int. J. Comp. Math, 81(12): $1519-1536$.

9. Fatunla, S.O., 1988. Numerical Methods for IVPs in ordinary differential equations.

Academic Press Inc. Harcourt Brace Jovanovich Publishers, New York

10. Jaun A. 2001. Numerical methods for partial differential equations. http: // pde. fusion.kth.se

11. Jacques, I. and C.J. Judd, 1987. Numerical Analysis. Chapman and Hall, N.York.

12. Kayode S.J. and D.O. Awoyemi, 2005. A 5-step maximal order method for direct solution of second order ordinary differential equations. J. Nig. Assoc. Math. Phys., 7: 285-292.

13. Liviu Gr. Ixaru, 1984. Numerical Methods for Differential Equations, Editura Academiei, Bucharest, Romania. 